\title{
SININHO: um serviço de mapeamento espaço-temporal para melhoria da tomada de decisão na gestão em saúde
}

\author{
Lindemberg Nascimento ${ }^{1}$, Renato Alves ${ }^{1}$, Daniel Victor Saraiva ${ }^{1}$, \\ Reinaldo Braga ${ }^{1}$, Carina Oliveira ${ }^{1}$ \\ ${ }^{1}$ Laboratório de Redes de Computadores e Sistemas (LAR) \\ Instituto Federal de Educação, Ciência e Tecnologia do Ceará (IFCE) \\ Caixa Postal 62.800-000 - Aracati - CE - Brazil
}

\begin{abstract}
Information and communication technologies have been providing innovative health solutions over the years. In this context, this paper presents a spatiotemporal mapping service for e-Health and m-Health systems for healthrelated data management. The proposal, called SININHO, was incorporated into a real health system for the diagnosis, treatment and follow-up of childhood and juvenile cancer. By optimizing spatiotemporal indicators, SININHO generates multiple strategic views of marker maps, density maps and heat maps. In this way, health managers will be able to analyze the presented scenarios and to base their decisions.
\end{abstract}

Resumo. As tecnologias da informação e comunicação vêm proporcionando soluções inovadoras em saúde ao longo dos anos. Neste contexto, este trabalho apresenta um serviço de mapeamento espaço-temporal para sistemas e-Healthe $m$-Health para gestão de dados relacionados à saúde. A proposta, denominada SININHO, foi incorporada em um sistema de saúde real de diagnóstico, tratamento e acompanhamento do câncer infanto-juvenil. Por meio da otimização de indicadores espaço-temporais, o SININHO gera múltiplas visualizações estratégicas de mapas de marcadores, mapas de densidade e mapas de calor (heat map). Dessa forma, gestores de saúde podem analisar os cenários apresentados e fundamentar as suas tomadas de decisões.

\section{Introdução}

O câncer em crianças e adolescentes não é uma única entidade patológica, mas um espectro de diferentes neoplasias malignas, que variam segundo tipo histológico, localização de origem da doença, sexo, idade e raça. A mortalidade por câncer em crianças e adolescentes em países desenvolvidos é considerada a segunda causa de morte na infância (crianças de 1 a 14 anos), correspondendo a cerca de 4\% a 5\% dos óbitos. Entretanto, nos países em desenvolvimento, essa proporção é bem menor, cerca de $1 \%{ }^{1}$. Nos adolescentes e adultos jovens (de 15 a 29 anos), o câncer é a principal causa de morte nos países desenvolvidos [INCA 2016].

No Brasil, os óbitos por câncer entre crianças, adolescentes e adultos jovens correspondem à segunda causa de morte. O câncer em crianças e adolescentes (de 0 a 19 anos) é considerado raro quando comparado com o câncer em adultos, correspondendo

\footnotetext{
${ }^{1}$ As principais causas de óbito na faixa etária de 1 a 14 anos ocorrem em razão das mortes por doenças infecciosas [INCA 2016].
} 
entre $2 \%$ e $3 \%$ de todos os tumores malignos registrados no país com taxas de mortalidade que variam entre 42,33 e 49,17 por milhão. A faixa etária que apresenta o maior risco de morte no país está no grupo de 15 a 19 anos (com 54,01 por milhão), especialmente para o sexo masculino (64,57 por milhão). Por outro lado, a faixa etária de menor risco está entre 5 a 9 anos. Além disso, em todas as faixas etárias, existe um predomínio nas taxas do sexo masculino, exceto para os menores de 1 ano [INCA 2016].

Para tentar reduzir esses índices de mortalidade, informação com qualidade é um recurso fundamental para chegar ao mais próximo da realidade, traçar suas características, detectar problemas e agir para solucioná-los. Muitos países em desenvolvimento adotaram sistemas eletrônicos de informação de gerenciamento de saúde para apoiar a gestão na prestação de serviços [Chikumba and Ramussen 2016]. Dessa forma, é essencial que a informação de saúde não seja apenas coletada, mas também analisada e disseminada entre as partes interessadas. Neste cenário, a informação, surge como o alicerce para o desenvolvimento do conhecimento tecnológico e científico para a inovação em saúde, sendo fundamental para guiar a gestão de serviços de saúde com eficiência, eficácia e efetividade.

Kanth et al. [Kanth et al. 2014] enfatizam que, nos últimos anos, as tecnologias da informação e comunicação têm desempenhado um papel vital em quase todos os setores do desenvolvimento humano, incluindo saúde, educação, transporte e desenvolvimento de infraestrutura. Os autores destacam que alguns dos impactos das tecnologias em sistemas de saúde atualmente têm os seguintes objetivos: melhorar a qualidade e a eficácia da saúde; ampliar as comunicações em tempo real entre os profissionais de saúde; aumentar a eficiência administrativa e dos processos de trabalho e diminuir a documentação e o tempo de trabalho improdutivo ou ocioso.

Visto que as informações são de extrema relevância para a tomada de decisões, o presente trabalho apresenta o SININHO, um sistema capaz de otimizar indicadores espaço-temporais de sistemas $e$-Health e $m$-Health para gestão de dados. Como estudo de caso, o SININHO foi incorporado ao SISAPP [Oliveira et al. 2017], um Sistema da Associação Peter Pan (APP) [APP 2018], entidade sem fins lucrativos que atende aproximadamente 2.200 pacientes, atuando na área da saúde voltada para o diagnóstico, tratamento e acompanhamento do câncer infanto-juvenil.

Por meio da otimização de indicadores espaço-temporais, o sistema SININHO gera múltiplas visualizações estratégicas de mapas de marcadores, mapas de densidade e mapas de calor (heat maps), possibilitando uma maior flexibilidade e visualização mais eficiente dos dados em comparação a utilização de formulários ou a consolidação de dados em tabelas. Desse modo, a principal questão que norteia esse sistema é a relevância dos mapas não apenas com uma estratégia de apresentação dos resultados, mas facilitando o estudo de correlações espaciais entre condições sociais, ambientais e a saúde, fazendo com que os profissionais de saúde tenham um maior direcionamento na tomada de decisões no planejamento, monitoramento e avaliação das ações de saúde.

O restante do trabalho está organizado da seguinte forma: a Seção 2 apresenta um referencial teórico destacando alguns trabalhos relacionados a ambientes de Sistemas de Informação Geográfica (SIGs); a Seção 3 detalha as fontes de informações do SININHO, desde a coleta de dados até sua integração com os mapas; na Seção 4 são ap- 
resentados os serviços de mapeamento espaço-temporais; e, por fim, na Seção 5, são destacadas as principais contribuições deste estudo e apresentadas as propostas futuras para o aperfeiçoamento e continuidade do mesmo.

\section{Trabalhos Relacionados}

Sistemas de informação em saúde são compreendidos como um conjunto de componentes integrados que atuam com o objetivo de obter dados e transformá-los em informações úteis para que os problemas de saúde de uma população possam ser identificados e consequentemente resolvidos. Nesse contexto, os trabalhos a seguir apresentam um referencial teórico de algumas pesquisas relacionadas aos ambientes de Sistemas de Informação Geográfica (SIGs) no mesmo contexto desta proposta.

No trabalho de [Freifeld et al. 2008], os autores apresentam um mapeamento global de alertas de doenças. O sistema desenvolvido por eles tem por objetivo aumentar a vigilância de doenças infecciosas por meio da integração de um conjunto de dados, provenientes da Organização Mundial da Saúde (OMS), ProMED-mail e de outras fontes disponíveis na Internet. Através do cruzamento desses dados, a aplicação obtém uma maior utilidade, já que analisá-los em conjunto é mais eficiente do que analisá-los individualmente. Por meio de um sistema automatizado de processamento de texto, os dados são separados por doença e exibidos por localidade, fornecendo, assim, um ponto de partida para a informação em tempo real sobre doenças infecciosas emergentes.

Um sistema de vigilância em tempo real de doenças transmitidas pela água, na cidade de Ahmedabad, na Índia, é apresentado em [Hughes et al. 2014]. O sistema proposto utiliza várias tecnologias, desde a coleta dos dados, casos de doenças geocodificadas, utilizando tablets Android, até sua análise, usando o Pysal. Esta última tecnologia é um framework baseado em Python que realiza uma segmentação de bases de dados em camadas Keyhole Markup Language (KML), cujos resultados são armazenados na nuvem para auxiliar a visualização de mapas, por meio do OpenStreetMap (OSM) [OSM 2018] .

No trabalho [Fei et al. 2016], os autores propõem uma relação espaço-temporal dos casos de câncer de tireoide e de mama na cidade de Hangzhou, na China. Eles utilizam o critério integrativo de previsibilidade da doença para observar a relação entre os dois tipos de câncer nas bases de conhecimento que mapeiam a doença. Após isso, são geradas previsões de incidência espaço-temporais de ambas doenças em dada região. Assim sendo, é realizada uma avaliação da variação espaço-temporal da incidência da doença para melhorar a compreensão das associações entre câncer de tireoide e de mama no nível populacional por meio da combinação da teoria da Entropia Máxima Bayesiana.

Bezerra et al. [Bezerra et al. 2017] desenvolveram um sistema denominado DENGOSA, um sistema Web de Informação Geográfica na área da epidemiologia capaz de fornecer serviços para controle de epidemias. O sistema complementa o ciclo epidemiológico fornecendo uma alimentação de qualidade à base do sistema federal por meio da integração das funcionalidades de coleta informatizadas do DENGOSA, suprindo a burocracia no processo de coleta de dados em fichas de papel à nível municipal. A fim de oferecer uma apresentação amigável dos dados coletados para as análises epidemiológicas no DENGOSA, foi projetado o uso de mapas Web com a geovisualização da incidência da epidemia.

Diante dos trabalhos citados, nota-se que os SIGs são excelentes ferramentas 
para combinar dados de diferentes fontes e apresentar informações de apoio à tomada de decisões. No entanto, o trabalho aqui proposto possui como diferencial em relação aos citados a geração de múltiplas visualizações estratégicas de mapas, como mapa de marcadores, mapas de densidade e mapas de calor (heat maps), identificando padrões, relações e anomalias de difícil detecção quando os dados são analisados de forma independente.

\section{Fluxo de Dados do Estudo de Caso}

Nesta seção, são apresentados os componentes e interações do SININHO, desde a coleta de dados até a geovisualização necessária para a geração dos mapas.

\subsection{Coleta de Dados}

A avaliação da incidência, da mortalidade e da morbidade hospitalar é importante para o delineamento do perfil de câncer e a efetiva vigilância para transformação de informações em ações efetivas para o controle do câncer. A coleta de dados aplicada nesse estudo é proveniente de duas bases de dados.

A primeira base provém do SISAPP [Oliveira et al. 2017], sistema web utilizado pela Associação Peter Pan (APP), ao qual o SININHO encontra-se incorporado. A base do SISAPP contém dados de incidência, tratamento e mortalidade do câncer infanto-juvenil, juntamente com sintomas, histórico familiar de câncer, tempo de diagnóstico (precoce, intermediário ou tardio) entre outras. No caso da base de dados do SISAPP, um paciente pode ser associado a três pontos geográficos distintos: município onde nasceu, município de procedência (local onde o paciente residia quando a doença foi diagnosticada) e o endereço atual (local onde o paciente reside durante o tratamento).

Além disso, o sistema SISAPP apresenta em seu banco de dados uma modelagem que proporciona a interação com informações geográficas onde os municípios cadastrados são referenciados com dados de latitude e longitude. Assim, no ato do cadastro da cidade natal de um paciente é realizada uma busca via Web Service das coordenadas correspondentes àquela cidade, as quais são utilizadas para o preenchimento dos mapas.

As taxas de mortalidade representam um importante subsídio para análise dos indicadores de saúde, mesmo nos países desenvolvidos e industrializados. Por meio da análise dessas taxas é possível medir o progresso na luta contra o câncer, bem como considerá-la um indicador do acesso ao tratamento da saúde no país [INCA 2008]. As informações sobre mortalidade, obtidas por meio do recolhimento sistemático das informações dos atestados de óbito, são as mais simples e acessíveis para o estudo das condições de saúde de uma população [INCA 2016].

Dessa forma, a segunda base provém das informações sobre mortalidade divulgadas pelo Sistema de Informação sobre Mortalidade (SIM), do Ministério da Saúde. A base do SIM engloba informações sobre todas as mortes ocorridas em território nacional, entre elas, causa da morte, local e data do óbito, cidade natal do indivíduo. Vale destacar que, pelo fato da Associação Peter Pan direcionar seus esforços à causa do câncer infantojuvenil, os dados do SIM que estão sendo utilizados no SININHO se referem apenas às crianças e adolescentes com idades entre 0 e 19 anos que foram acometidas com câncer. 


\subsection{Extração e Transformação dos Dados}

O principal objetivo da extração é adquirir os dados necessários e prepará-los para gerar uma base de dados separada que proporcione uma análise de acordo com as principais necessidades da gestão. A extração auxilia no descarte de inconsistências da base de dados, tratando os valores nulos e eliminando registros irrelevantes.

Após a extração, os dados passam por um processo de transformação em um formato aberto como serviço. Para a troca dos dados entre o sistema e o serviço, é utilizado o formato JavaScript Object Notation (JSON), que tem capacidade de estruturar informações de forma mais compacta e legível, tornando, assim, mais fácil a análise de tais dados.

\subsection{Geovisualização}

Após concluída a coleta, extração e transformação, o próximo passo é a geovisualização. Nessa etapa, foi utilizada a Leaflet, uma biblioteca JavaScript de código aberto, que permite a construção de mapas interativos em projetos Web de maneira simples, com alta performance e usabilidade.

Através do framework OpenStreetMap (OSM) - um software livre de código aberto [OSM 2018], a Leaflet tem acesso à uma coleção de imagens cartográficas de mapas, baseadas em dados Raster. Com os dados do OSM, um usuário está livre para fazer o download de qualquer mapa que deseje, permitindo o uso dos dados off-line. Esse processo de geovisualização tem como principal objetivo auxiliar o aprimoramento da assistência ao paciente, a pesquisa de base clínica e também prover subsídios às atividades administrativas.

\section{Sistema SININHO}

Esta seção aborda detalhes do SININHO, um serviço baseado em localização (LocationBased Services - LBS) capaz de gerar múltiplas visualizações estratégicas para o apoio à tomada de decisão na saúde através de mecanismos de geovisualização. No estudo de caso proposto, o SININHO aparece como uma nova camada de serviços, com o objetivo de gerar informações enriquecidas a partir de dados provenientes do SISAPP e dados do Sistema de Informações sobre Mortalidade (SIM). O serviço apresenta três visualizações distintas de mapas: (i) mapa de marcadores; (ii) mapa de densidade e (iii) mapa de calor. Em cada uma das visualizações há uma série de filtros que possibilitam aos gestores e profissionais de saúde uma visão a nível macro e micro de determinadas situações.

\subsection{Mapa de Marcadores}

A Figura 1 apresenta um exemplo de Mapa de Marcadores (MM) (com a logomarca da APP). O mapa apresenta os municípios com pelo menos um paciente atendido pela associação de acordo com o filtro aplicado, onde cada marcador corresponde ao total de pacientes diagnosticados com câncer em uma determinada cidade ${ }^{2}$. Já a Figura 2 apresenta marcadores de dados reais de mortalidade por câncer com foco em crianças e

\footnotetext{
${ }^{2}$ Para não comprometer a privacidade da instituição e de seus pacientes, os dados aqui apresentados em relação à quantidade de pacientes e municípios não são reais. Vale destacar também que a Associação Peter Pan está localizada no Ceará, ou seja, a maior quantidade de seus pacientes é de procedência da região nordeste, principalmente, do estado do Ceará. Por isso, as imagens apresentadas destacam essa região.
} 
adolescentes com idades entre 0 a 19 anos em 2014 que provêm das informações sobre mortalidade divulgadas no SIM em municípios com pelo menos um óbito.

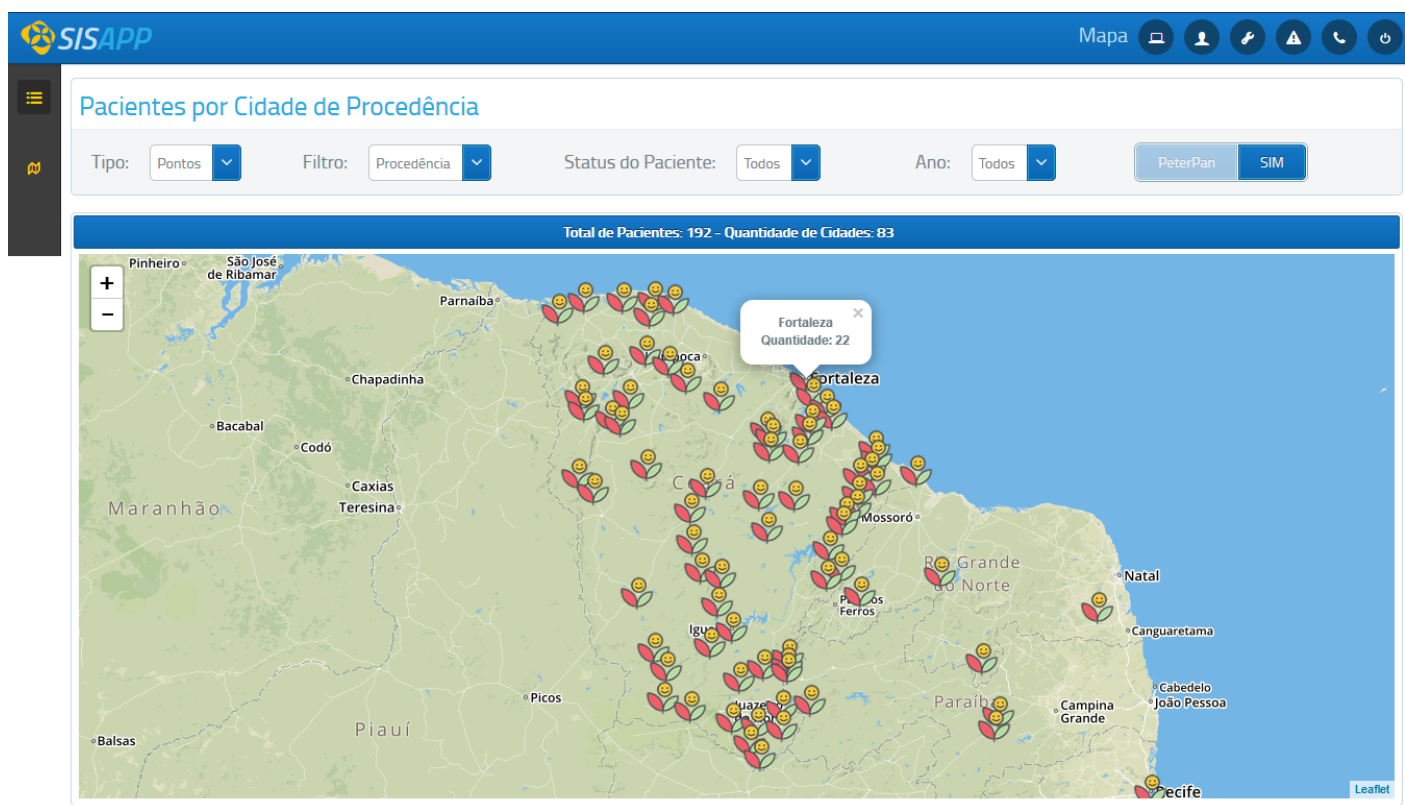

Figure 1. Exemplo de Mapa de Marcadores com pacientes por cidade de procedência com dados do SISAPP.

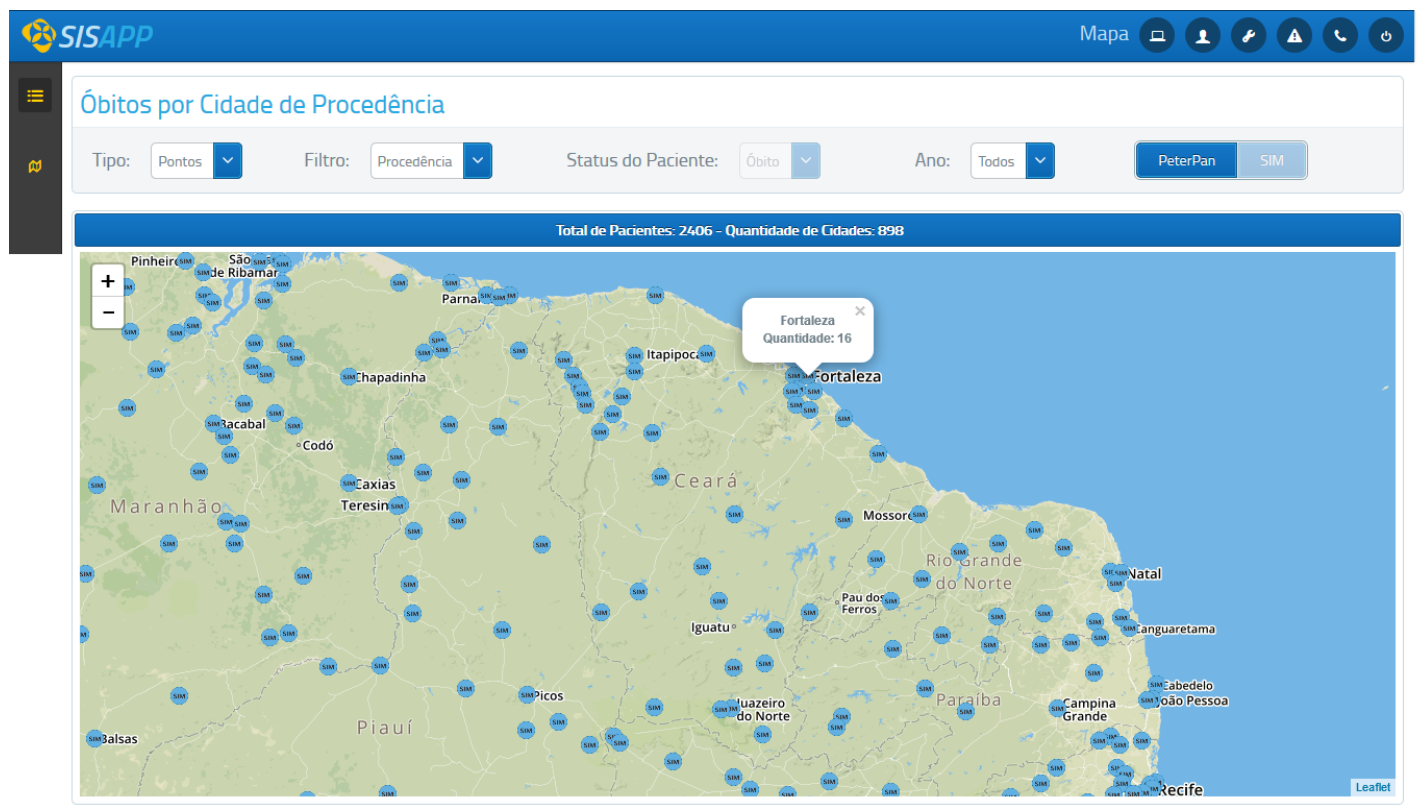

Figure 2. Exemplo de Mapa de Marcadores com óbitos por cidade de procedência com dados do SIM.

Conforme ilustrado no topo das figuras, os filtros que podem ser aplicados são referentes aos seguintes indicadores: procedência dos pacientes (onde mora ou morava); naturalidade dos pacientes (cidade natal); pacientes que foram a óbito e municípios que receberam treinamento. Em relação ao Status dos Pacientes, as escolhas possíveis são: em análise; em tratamento; curado, óbito, abandono, dentre outras. As demais opções 
estão relacionadas a escolha do ano a ser pesquisado e a possibilidade de alternar entre as bases de dados da associação e do SIM.

Em vista disso, os gestores e profissionais de saúde podem facilmente buscar, por exemplo, a quantidade de crianças que vieram a óbito em um determinado ano, procedentes e/ou naturais de determinada cidade.

\subsection{Mapa de Calor}

A visualização de um Mapa de Calor (MC) consiste em um cálculo de densidade de pontos em dada região e, em sequência, a aplicação de uma cor (a partir de uma gama de cores predefinidas para esta região) [Meier and Heidmann 2014]. Tal visualização permite um entendimento rápido da situação e do impacto causado por ela, mesmo com um grande número de elementos apresentados de uma só vez. Este tipo de mapa faz parte de um plug-in da API Leaflet e funciona como uma camada sobreposta ao mapa padrão.

A Figura 3 apresenta, o que seria no Mapa de Calor, a naturalidade dos pacientes atendidos na associação. Além disso, a Figura 4 apresenta a visualização de parte da região Nordeste do Brasil em 2014. Para essa figura, são utilizados dados reais de mortalidade por câncer com foco em crianças e adolescentes da base de dados do SIM, que neste caso, também leva-se em consideração os endereços de naturalidade dos indivíduos.

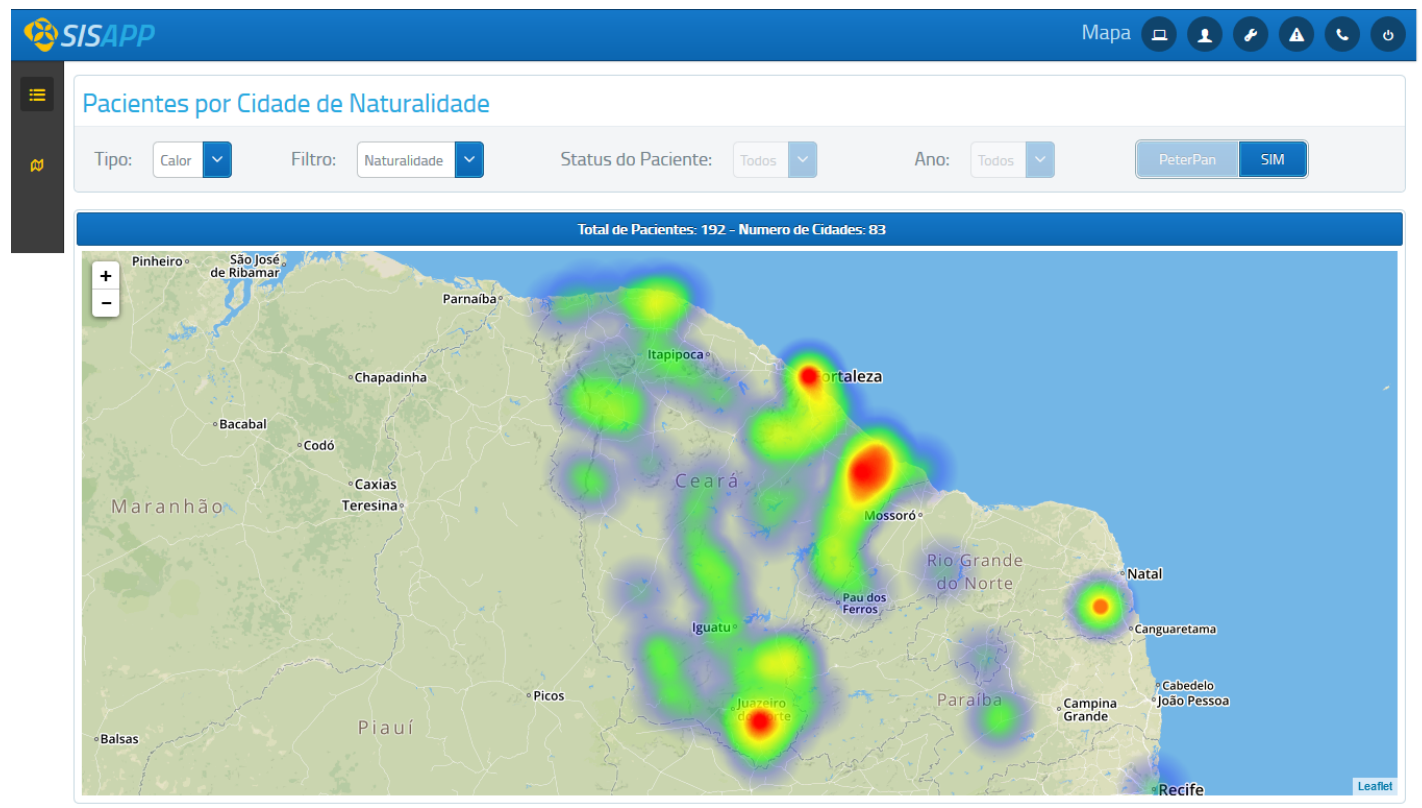

Figure 3. Exemplo de Mapa de Calor com pacientes atendidos pela associação.

Nota-se que o MC apresenta uma abordagem bem diferente da ilustrada no mapa de marcadores. No MC é possível perceber um padrão, onde há um maior número de ocorrências nas capitais e regiões metropolitanas dos estados mostrados. A partir desses padrões é possível, por exemplo, direcionar treinamentos e campanhas publicitárias, além de estudos epidemiológicos para analisar as correlações e causas de incidência nos municípios analisados. 


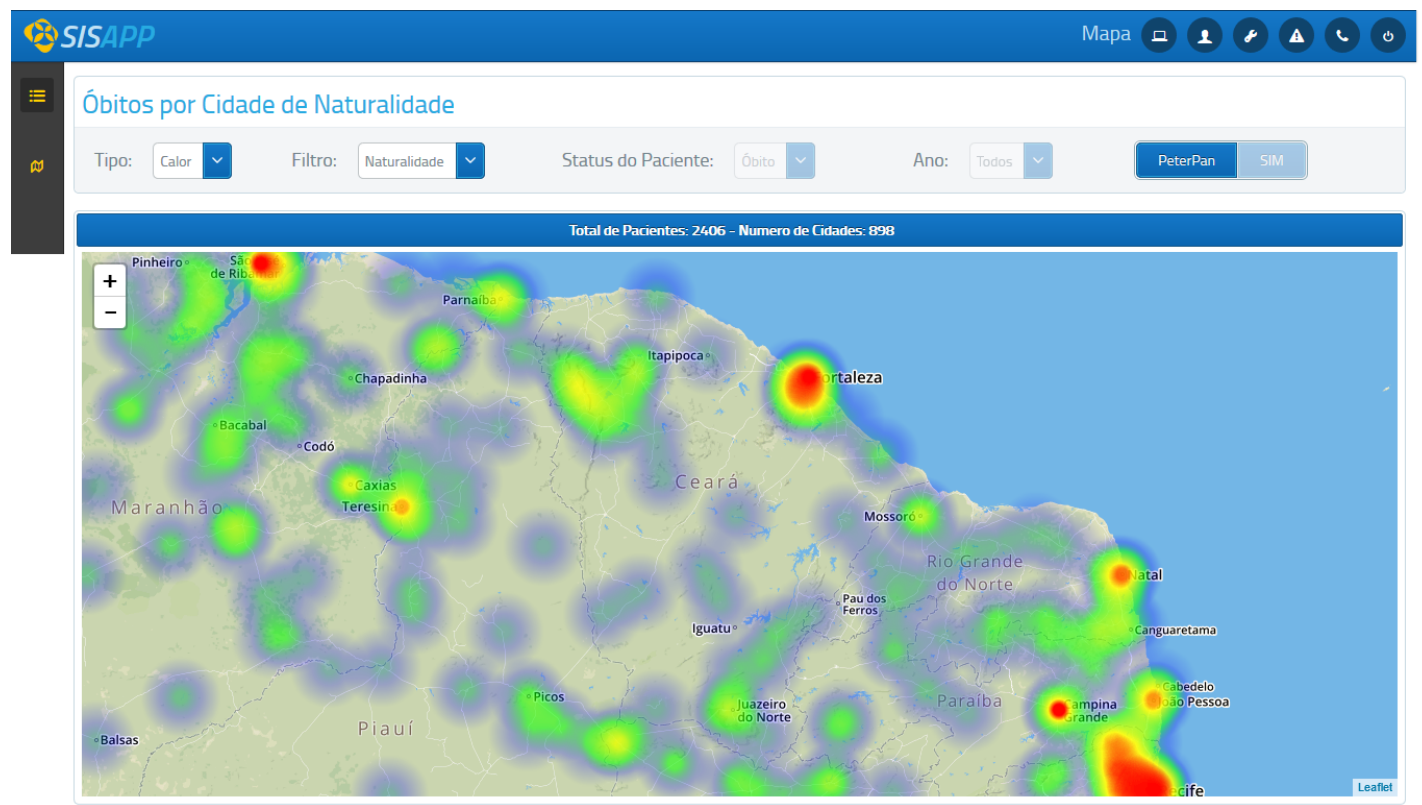

Figure 4. Exemplo de Mapa de Calor com Óbitos por Naturalidade na Região Nordeste do Brasil.

\subsection{Mapa de Densidade}

O serviço de Mapa de Densidade (MD) disponibiliza uma visualização mais refinada de cada município. Neste caso, utiliza-se a demarcação estabelecida pelo Instituto Brasileiro de Geografia e Estatística (IBGE).

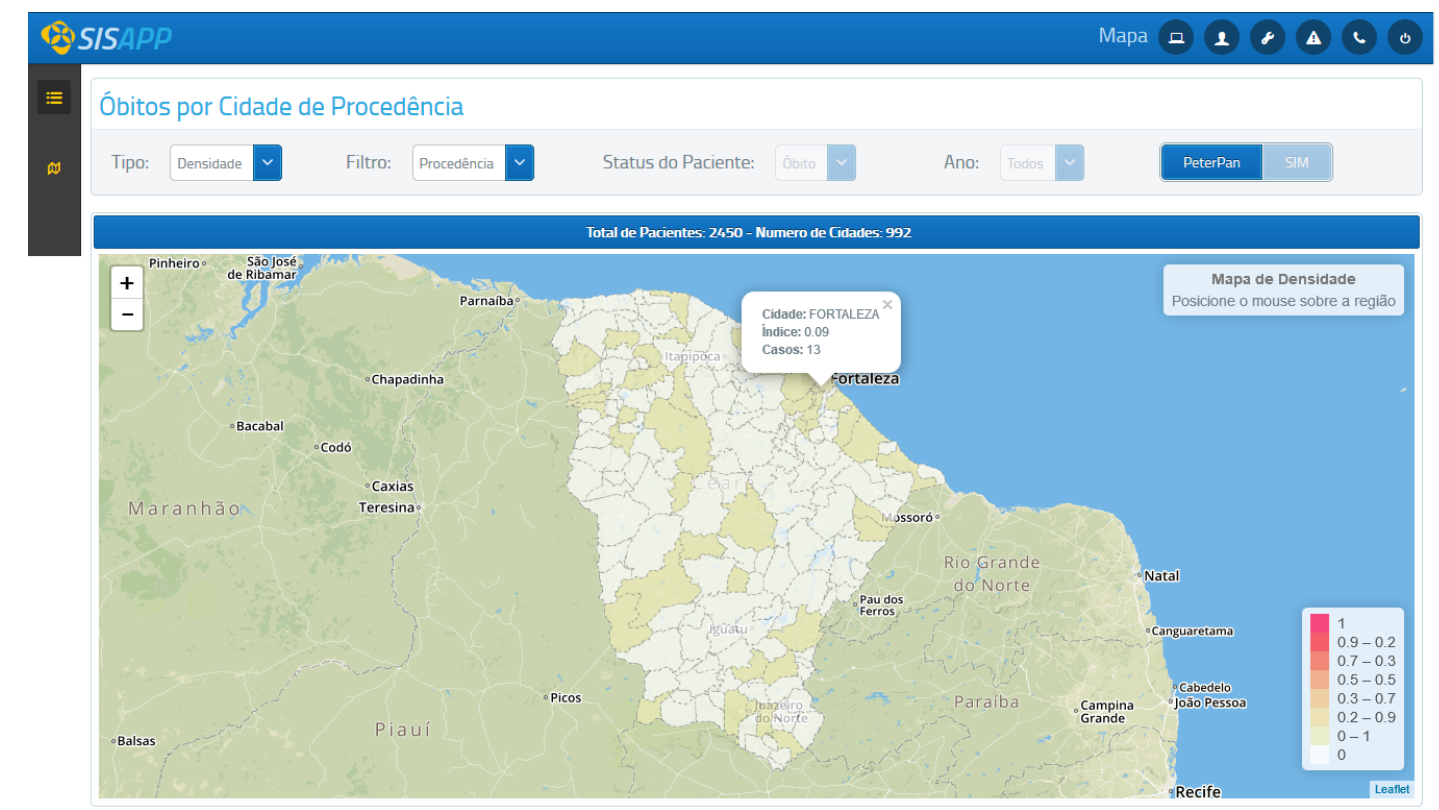

Figure 5. Exemplo de Mapa de Densidade com óbitos por procedência no Estado do Ceará.

A Figura 5 apresenta um exemplo de mapa de densidade utilizando os dados do Sistema de Informações sobre Mortalidade, que, assim como no mapa de calor referente aos dados do SIM, são reais. Nesse caso, são apresentados os óbitos por cidade de 
procedência, no estado do Ceará em 2014. Em destaque, podemos observar uma caixa de texto mostrando a cidade selecionada (Fortaleza), a quantidade de casos ocorridos no local (13 casos) e o índice percentual das ocorrências daquele município $(0,09)$ em relação ao total de casos no país.

O serviço do mapa de densidade permite uma visão mais aprofundada, já que delimita geograficamente cada cidade e possibilita, no caso dos dados do SIM, a identificação de áreas com maior ocorrência de óbitos onde as regiões com cores mais escuras apresentam maiores índices de óbitos e as regiões mais claras, consequentemente, apresentam menores índices.

\section{Conclusões}

Nos últimos anos, os principais sistemas informativos vêm sendo incorporados, majoritariamente, às tecnologias da informação e comunicação. Neste contexto, é importante que a área da saúde passe por um processo de modernização de suas metodologias, abrindo espaço para que as tecnologias se tornem, efetivamente, sua grande aliada no processo de gestão. Dentre os benefícios, em relação aos métodos que não utilizam a informática, podemos destacar maior agilidade, interatividade e facilidade de visualização dos dados. Com a utilização de formulários de papel, por exemplo, a gestão não tem como manusear grandes quantidades de dados de forma ágil e fácil a ponto de convertê-los em informações concisas e coesas.

Entretanto, por meio de interfaces gráficas, obtém-se uma visualização mais efetiva dos dados, além de uma maior flexibilidade. Em vista disso, ao serem agregados à gestão de saúde, por intermédio do SININHO, os três modos de mapeamento propostos geram uma melhora técnico-administrativa considerável na gestão de dados sobre saúde. O serviço permite aos gestores e profissionais da área realizar consultas de forma intuitiva e amigável por intermédio de uma interface compreensível. Desta maneira, a gestão consegue identificar, de maneira ágil, aspectos que podem subsidiar programas de prevenção e diagnóstico precoce do câncer infanto-juvenil, por exemplo.

É importante destacar que o SININHO pode ser utilizado de forma independente ou anexo a sistemas de $e$-Health ou $m$-Health (como é o caso do SISAPP). Por isso, através do serviço de mapeamento espaço-temporal aqui proposto, é possível atender, de imediato, a demanda de outras entidades de saúde devido sua grande capacidade de adaptação uma vez que a automatização do processo de geração de mapas estratégicos é claramente vantajosa, em especial quando a disponibilidade da informação para a tomada de decisão afeta a qualidade de vida de pessoas com câncer ou de outras doenças como diabetes, AIDS, cólera, dengue, entre outras principais do Brasil.

Como trabalhos futuros para o SININHO, almeja-se a adição de novos filtros, permitindo uma visão mais detalhada de cenários críticos em saúde e a integração com Machine Learning, utilizando Aprendizado Não Supervisionado, visando a Clusterização dos dados. Isso vai ajudar a gestão no processo de identificação de padrões. Por exemplo, é possível identificar que determinado tipo de doença é mais recorrente em crianças em uma determinada faixa de idade que já viveram em uma dada região. Com a agregação dessas novas ferramentas ao SININHO, espera-se que a gestão de saúde obtenha uma eficiência ainda maior em suas atividades e que, assim, eleve o índice de cura e melhore a qualidade de vida de crianças e adolescentes com câncer e de suas famílias. 


\section{References}

APP (2018). Associação Peter Pan (APP). http://www.app.org.br. [Último Acesso em: 15-Março-2018].

Bezerra, A., Bento Filho, J. J., Braga, R. B., Oliveira, C. T., and Oliveira, A. M. B. (2017). Dengosa: Um sistema de informação geográfica para apoio à decisão no controle de epidemias. In XVI Workshop de Ferramentas e Aplicações (WFA)/Simpósio Brasileiro de Sistemas Multimídia e Web (WebMedia), pages 179-183.

Chikumba, P. A. and Ramussen, S. L. (2016). Management and use of health information in malawi and burkina faso: The role of technology. In IST-Africa Week Conference, pages $1-9$.

Fei, X., Christakos, G., Lou, Z., Lou, Z., Liu, Q., and Wu, J. (2016). Spatiotemporal co-existence of female thyroid and breast cancers in hangzhou. Scientific Reports, 6.

Freifeld, C. C., Mandl, K. D., Reis, B. Y., and Brownstein, J. S. (2008). Healthmap: Global infectious disease monitoring through automated classification and visualization of internet media reports. Journal of the American Medical Informatics Association, 15(2):150-157.

Hughes, C., Naik, V. S., Sengupta, R., and Saxena, D. (2014). Geovisualization for Cluster Detection of Hepatitis A and E Outbreaks in Ahmedabad, Gujarat, India. In ACM SIGSPATIAL International Workshop on the Use of GIS in Public Health, HealthGIS, pages 39-44, New York, NY, USA. ACM.

INCA (2008). Câncer da criança e adolescente no Brasil: dados dos registros de base populacional e de mortalidade. Rio de Janeiro: INCA.

INCA (2016). Incidência, mortalidade e morbidade hospitalar por câncer em crianças, adolescentes e adultos jovens no Brasil: informações dos registros de câncer e do sistema de mortalidade. Rio de Janeiro: INCA.

Kanth, R. K., Liljeberg, P., Westerlund, T., Kumar, H., Tenhunen, H., Wan, Q., Yang, G., and Zheng, L. R. (2014). Information and communication system technology's impacts on personalized and pervasive healthcare: A technological survey. In IEEE Conference on Norbert Wiener in the 21st Century (21CW), pages 1-5.

Meier, S. and Heidmann, F. (2014). Too many markers, revisited: An empirical analysis of web-based methods for overcoming the problem of too many markers in zoomable mapping applications. In International Conference on Computational Science and Its Applications, pages 121-125.

Oliveira, R. A., Nascimento Filho, L. B., Vieira, W. O., Bandeira, T. F. L., Oliveira, A. M. B., Braga, R. B., and Oliveira, C. T. (2017). Sistema de apoio à tomada de decisão na gestão de atendimento a pacientes com câncer infanto-juvenil. In XVI Workshop de Ferramentas e Aplicações (WFA)/Simpósio Brasileiro de Sistemas Multimídia e Web (WebMedia), pages 189-193.

OSM (2018). OpenStreetMap. http://www.openstreetmap.org/. [Último Acesso em: 15-Março-2018]. 